\title{
Singularities and moments of nonlinear systems
}

\author{
Alberto Padoan, Member, IEEE, and Alessandro Astolfi, Fellow, IEEE
}

\begin{abstract}
The notions of eigenvalue, pole and moment at a pole of a continuous-time, nonlinear, time-invariant system are studied. Eigenvalues and poles are first characterized in terms of invariant subspaces. Tools from geometric control theory are used to define nonlinear enhancements of these notions and to study their relationship with the solution of certain partial differential equations, cascade decompositions and steady-state impulse responses. The theory is illustrated by means of worked-out examples and its significance is demonstrated by solving the model reduction problem by moment matching at poles for nonlinear systems.
\end{abstract}

Index Terms-Eigenvalues, nonlinear systems, modeling, model reduction

\section{INTRODUCTION}

The goal of this work is to develop a nonlinear enhancement of the notions of eigenvalue, pole and moment at a pole of a continuoustime, linear, time-invariant system. The interest in these notions is motivated by the following observation. The paper [1] has shown that the moments of a system at an ordinary point 1 uniquely determine the steady-state response of the system under the effect of specific inputs, provided that some assumptions hold. Similarly, the paper [3] has shown that the moments of a linear system at a pole uniquely specify the steady-state impulse response of the system (or, equivalently, the free response of the system for a specific initial condition). For linear systems, reduced order models computed by matching the steady-state impulse response can thus be used to approximate resonant behaviors, as they approximate the behavior of a system near a singularity of the transfer function. For nonlinear systems, the approximation of resonant behaviors by moment matching thus hinges upon finding appropriate nonlinear counterparts of the notions of eigenvalue, pole and moment at a pole.

The literature abounds with nonlinear counterparts of the notions of eigenvalue and pole. For linear systems, these notions are fundamental tools for analysis and design [4-9] and for the solution of a wide range of linear and nonlinear control-theoretic problems [4-12]. While it is out of the scope of this paper to provide a comprehensive overview, we emphasise that several notions of eigenvalues and poles for nonlinear systems have appeared in the literature recently, e.g., in [13-21]. For example, an algebraic approach has been used to define a notion of eigenvalue and to study the pole-zero structure of a nonlinear system in $[13,14]$. For linear time-varying systems, eigenvalues and poles have been defined, e.g., in $[15,16]$. In $[17,18]$, the Koopman operator has been exploited to analyse spectral properties of nonlinear dynamical systems. Motivated by applications to stability, observability

This work has been partially supported by the European Union's Horizon 2020 Research and Innovation Programme under grant agreement No 739551 (KIOS CoE).

A. Padoan is with the Department of Engineering, University of Cambridge, Cambridge CB2 1PZ, UK (email: a.padoandeng. cam.ac.uk).

A. Astolft Is with the Department of Electrical and Electronic Engineering, Imperial College London, London SW7 2AZ, UK and with the Dipartimento di Ingegneria Civile e Ingegneria Informatica, Università di Roma "Tor Vergata", Via del Politecnico 1, 00133, Rome, Italy (email: a.astolfi@imperial.ac.uk.

${ }^{\text {I }}$ The terminology is borrowed from $[2, \mathrm{p} .861]$, where a point $\bar{s} \in \mathbb{C}$ is said to be an ordinary point of a transfer function if the transfer function is analytic in a neighborhood of $\bar{s}$. and contraction analysis, a different notion of eigenvalue for nonlinear systems has been recently investigated in [19-21].

This work builds on preliminary results first obtained in [3, 22, 23]. The present paper reorganizes our preliminary findings in a systematic fashion and provides detailed proofs of previous results. The starting point of our analysis is the notion of moment at a pole of a linear system introduced in [3], which has been shown to be in oneto-one correspondence with the solution of a "reduced" Sylvester equation [22]. Analogous results are obtained in this paper for nonlinear systems by defining nonlinear counterparts of the notions of eigenvalue, pole and moment at a pole. The key ingredients of our analysis are the novel notions of $f$-reducing manifold and of $f$ reducing distribution, which together provide a nonlinear counterpart of the notion of $A$-reducing subspace [24]. The significance of these notions is demonstrated by a model reduction framework which unifies the approach of moment matching and that of modal approximation [25, Chapter 9]. The theory is illustrated by means of worked-out examples and by approximating forced oscillations in a system.

The remainder of this work is organized as follows. Section II provides basic definitions and preliminary results. Section III contains the main results of the paper. Section IV develops a model reduction method by moment matching at poles for nonlinear systems. Section $\mathrm{V}$ concludes the paper with a summary of the results and an outlook to future research.

Notation: The notation is standard and mostly borrowed from $[7,10,11] . \mathbb{Z}, \mathbb{R}$ and $\mathbb{C}$ denote the set of integer numbers, the set of real numbers, and the set of complex numbers, respectively. The subscripts + and - are used to indicate the non-negative elements (or the elements with non-negative real part) and the negative elements (or the elements with negative real part) of a set. $e_{j}$ denotes the vector with the $j$-th entry equal to one and all other entries equal to zero. $I$ denotes the identity matrix. $M^{\prime}$ denotes the transpose of the matrix $M \in \mathbb{C}^{p \times m} \cdot \sigma(A)$ denotes the spectrum of the matrix $A \in \mathbb{C}^{n \times n}$. A matrix is non-derogatory if its characteristic and minimal polynomials coincide [26, p.77]. $\|x\|$ denotes the Euclidean norm of the vector $x \in \mathbb{C}^{n}$. Given $A \in \mathbb{C}^{n \times n}$, a subspace $\mathcal{S}$ of $\mathbb{C}^{n}$ is $A$-invariant if $x \in \mathcal{S}$ implies $A x \in \mathcal{S}$ [24, p. 143] and is $A$-reducing if $\mathcal{S}$ is $A$-invariant and there exists an $A$-invariant subspace $\mathcal{T}$ of $\mathbb{C}^{n}$ such that $\mathcal{T} \oplus \mathcal{S}=\mathbb{C}^{n}$ [24, p.197], in which case the pair of subspaces $\mathcal{S}$ and $\mathcal{T}$ is also said to be $A$-reducing. The generalized eigenspace of $A \in \mathbb{C}^{n \times n}$ associated with the eigenvalue $s^{\star} \in \sigma(A)$ is the subspace $\mathcal{E}_{s^{\star}}=\operatorname{Ker}\left(A-s^{\star} I\right)^{m}$, with $m \in \mathbb{Z}_{+}$such that $\operatorname{Ker}\left(A-s^{\star} I\right)^{m-1} \neq \operatorname{Ker}\left(A-s^{\star} I\right)^{m}=\operatorname{Ker}\left(A-s^{\star} I\right)^{m+1}$. $\delta_{0}$ denotes the Dirac $\delta$-function [8, p.16].

\section{Preliminaries}

Consider a continuous-time, single-input, single-output, linear, timeinvariant system described by the equation 2

$$
\dot{x}=A x+B u, \quad y=C x,
$$

${ }^{2}$ We consider complex-valued linear systems to simplify the exposition. Analogous results can be deduced for real-valued systems using arguments similar to those in [1]. Note also that the time argument is omitted when possible. 
in which $x(t) \in \mathbb{C}^{n}, u(t) \in \mathbb{C}, y(t) \in \mathbb{C}$ and $A \in \mathbb{C}^{n \times n}, B \in \mathbb{C}^{n \times 1}$ and $C \in \mathbb{C}^{1 \times n}$ are constant matrices, respectively. Throughout the paper we assume that system (1) is minimal, i.e. reachable and observable, and define its transfer function as $W(s)=C(s I-A)^{-1} B$.

This section recalls basic properties of $A$-reducing subspaces and their relationship with generalized eigenspaces, cascade decompositions and moments at a pole of a system [3, 22, 23].

Lemma 1. [24, p.198] Let $A \in \mathbb{C}^{n \times n}$. The subspaces $\mathcal{S}$ and $\mathcal{T}$ are $A$-reducing if and only if $A \widetilde{\Pi}=\widetilde{\Pi} A$, with $\widetilde{\Pi}$ the projector onto $\mathcal{S}$ along $\mathcal{T}$.

Lemma 2. [23] Consider system (1) and let $s^{\star} \in \sigma(A)$. Then the generalized eigenspace $\mathcal{E}_{S^{\star}}$ is the largest $A$-reducing subspace $\mathcal{S}$ of $\mathbb{C}^{n}$ such that $\sigma\left(\left.A\right|_{\mathcal{S}}\right)=\left\{s^{\star}\right\}$, with $\left.A\right|_{\mathcal{S}}$ the restriction of $A$ to $\mathcal{S}$.

Lemma 3. [23] System (1) has a pole at $s^{\star} \in \mathbb{C}$ of order $m<r$, with $r$ the relative degree of the transfer function of system (1), if and only if $\operatorname{dim} \mathcal{E}_{s^{\star}}=m$ and there exist $\Theta \in \mathbb{C}^{n \times 1}$ and $\Gamma \in \mathbb{C}^{\Gamma \times n}$ such that (i) $B$ is the projection of $\Theta$ onto $\mathcal{E}_{s^{\star}}$, (ii) $C$ is the projection of $\Gamma$ onto $\mathcal{E}_{s^{\star}}^{\perp}$, (iii) $\mathcal{E}_{s^{\star}}$ is $(A-\Theta \Gamma)$-invariant.

Definition 1. [3] Consider system (1) and let $s^{\star} \in \mathbb{C}$ be a pole of order $m \in \mathbb{Z}_{+}$of its transfer function. The moment of order $-k$ of system (1) at $s^{\star}$ is defined as the complex number

$$
\eta_{-k}\left(s^{\star}\right)=\frac{1}{(m-k) !}\left[\frac{d^{m-k}}{d s^{m-k}}\left(s-s^{\star}\right)^{m} W(s)\right]_{s=s^{\star}}
$$

for every integer $1 \leq k \leq m$.

Theorem 1. [22] Consider system (1] and assume its transfer function has a pole of order $m \in \mathbb{Z}_{+}$at $s^{\star} \in \mathbb{C}$. Suppose

$$
A=\left[\begin{array}{cc}
A_{11} & A_{12} \\
0 & A_{22}
\end{array}\right], B=\left[\begin{array}{c}
0 \\
B_{2}
\end{array}\right], C=\left[\begin{array}{c}
C_{1}^{\prime} \\
0
\end{array}\right]^{\prime},
$$

in which $A_{11} \in \mathbb{C}^{(n-m) \times(n-m)}$ is such that $s^{\star} \notin \sigma\left(A_{11}\right)$, $A_{12} \in \mathbb{C}^{(n-m) \times m}$ is such that $\operatorname{rank} A_{12}=1$ and $A_{22} \in \mathbb{C}^{m \times m}$ is a non-derogatory matrix with characteristic polynomial

$$
\chi(s)=\left(s-s^{\star}\right)^{m} .
$$

Then there exists a one-to-one correspondence 3 between the moments $\eta_{-1}\left(s^{\star}\right), \eta_{-2}\left(s^{\star}\right), \ldots, \eta_{-m}\left(s^{\star}\right)$ and the matrix $C_{1} \Pi$, where $\Pi \in \mathbb{C}^{(n-m) \times m}$ is the unique solution of the reduced Sylvester equation

$$
A_{11} \Pi+A_{12}=\Pi A_{22} .
$$

Theorem 2. [22] Consider system (1) and assume its transfer function has a pole of order $m \in \mathbb{Z}_{+}$at $s^{\star} \in \mathbb{C}_{+}$. Suppose (3) holds and let $x=\operatorname{col}\left(x_{1}, x_{2}\right)$, with $x_{1} \in \mathbb{C}^{n-m}$ and $x_{2} \in \mathbb{C}^{m}$. Assume $A_{11} \in \mathbb{C}^{(n-m) \times(n-m)}$ is such that $\sigma\left(A_{11}\right) \subset \mathbb{C}_{-}$, $A_{12} \in \mathbb{C}^{(n-m) \times m}$ is such that $\operatorname{rank} A_{12}=1$ and $A_{22} \in \mathbb{C}^{m \times m}$ is a non-derogatory matrix with characteristic polynomial (4) such that the pair $\left(A_{22}, B_{2}\right)$ is reachable. Then there exists a one-to-one correspondence between the moments $\eta_{-1}\left(s^{\star}\right), \eta_{-2}\left(s^{\star}\right), \ldots, \eta_{-m}\left(s^{\star}\right)$ and the steady-state impulse response of the output of system 1 .

\section{MAIN RESULTS}

This section extends the results in the previous section to continuoustime, single-input, single-output, nonlinear, time-invariant systems described by the equations 4

$$
\dot{x}=f(x)+g(x) u, \quad y=h(x),
$$

\footnotetext{
${ }^{3}$ Throughout this work by one-to-one correspondence between two objects we mean that one uniquely determines the other and vice versa.

${ }^{4}$ The choice of focusing on input-affine systems is made to ensure that the steady-state impulse response of the system, and its derivatives, are well-defined (see $[1,27]$ for more detail).
}

in which $x(t) \in \mathbb{R}^{n}, \quad u(t) \in \mathbb{R}, \quad y(t) \in \mathbb{R}$ and the mappings $f: \mathbb{R}^{n} \rightarrow \mathbb{R}^{n}, g: \mathbb{R}^{n} \rightarrow \mathbb{R}^{n}, h: \mathbb{R}^{n} \rightarrow \mathbb{R}$ are such that $f(0)=0$ and $h(0)=0$, respectively. All mappings are assumed to be smooth, i.e. infinitely many times differentiable, and system (6) is assumed to be minimal, i.e. strongly observable and accessible [11, Chapter 3].

\section{A. Eigenvalues and invariance notions}

Definition 2. Consider system (6). The system

$$
\dot{\omega}=s(\omega),
$$

with $\omega(t) \in \mathbb{R}^{m}$ and $s: \mathbb{R}^{m} \rightarrow \mathbb{R}^{m}$ such that $s(0)=0$, is said to be immersed into system $\left(6\right.$ if there exists a mapping $\tilde{\pi}: \mathbb{R}^{m} \rightarrow \mathbb{R}^{n}$, locally defined in a neighborhood of the origin and such that $\tilde{\pi}(0)=0$, which solves the partial differential equation

$$
\frac{\partial \widetilde{\pi}}{\partial \omega} s(\omega)=f \circ \tilde{\pi}(\omega) .
$$

Definition 2 is a modification of the notion of immersion given in [10]. This property can be intuitively interpreted as a notion of "projection" of a system onto another system and has been used for diverse applications in control theory $[10,11,28]$. Note that Definition 2 formalizes a property of systems described only by an ordinary differential equation, while in [10] the notion of immersion is given for systems with outputs.

Definition 3. Consider system (6). The manifold

$$
\mathcal{M}=\left\{x \in \mathbb{R}^{n}: x=\tilde{\pi}(\omega), \omega \in \mathbb{R}^{m}\right\},
$$

with $\tilde{\pi}: \mathbb{R}^{m} \rightarrow \mathbb{R}^{n}$ such that $\tilde{\pi}(0)=0$, is said to be an $f$-reducing manifold if $f$ is tangent to $\mathcal{M}$ at the origin and the following hold.

(i) There exists a non-singular, involutive, $f$-invariant distribution $\Delta$ defined in a neighborhood $U$ of the origin.

(ii) For every $x \in U$ the condition $\Delta(x) \oplus T_{x} \mathcal{M}=T_{x} \mathbb{R}^{n}$ holds.

(iii) The restriction of system (6) to the manifold $\mathcal{M}$ is immersed into system (6), i.e. 8) holds for some $s$.

Definition 3 can be seen as a nonlinear counterpart of the notion of $A$-reducing pair of subspaces and can be interpreted geometrically as follows. Condition (i) guarantees the existence of a partition of every sufficiently small neighborhood of the origin into leaves of the foliation induced by the distribution $\Delta$. Condition (ii) is a transversality condition which ensures that the tangent space at the origin of the ambient manifold $\mathbb{R}^{n}$ is decomposed into the tangent space of the manifold $\mathcal{M}$ at the origin and the tangent space of the maximal integral submanifold $\mathcal{N}$ of $\Delta$ passing through the origin (see Figure 1). Finally, condition (iii) is an immersion condition which ensures that $\mathcal{M}$ is (locally) an invariant manifold.

Remark 1. While the notion of $A$-reducing subspace is inherently symmetric, the roles of the manifold $\mathcal{M}$ and of the distribution $\Delta$ in Definition 3 are not interchangeable. This is because the notions of invariance of a manifold and of a distribution do not coincide for nonlinear systems.

Any $f$-reducing manifold of system (6) can be also characterized as follows. Let $U$ be an open subset of $\mathbb{R}^{n}$ containing the origin and let $\Delta$ be a non-singular, involutive, $f$-invariant distribution on $U$. Then there exist (local) coordinates $x=\operatorname{col}\left(x_{1}, x_{2}\right)$, with $x_{1} \in \mathbb{R}^{n-m}$, $x_{2} \in \mathbb{R}^{m}$ and $n-m$ the dimension of $\Delta$ on $U$, in which the vector field $f$ reads as

$$
f(x)=\left[\begin{array}{c}
f_{1}\left(x_{1}, x_{2}\right) \\
f_{2}\left(x_{2}\right)
\end{array}\right]
$$

and every vector field $\theta \in \Delta$ is of the form $\theta(x)=\left[\theta_{1}(x)^{\prime} 0\right]^{\prime}$. Let the manifold $\mathcal{M}=\left\{x \in \mathbb{R}^{n}: x_{1}=\pi\left(x_{2}\right), x_{2} \in \mathbb{R}^{m}\right\}$ be tangent to $f$ at the origin. In the above coordinates, the transversality 


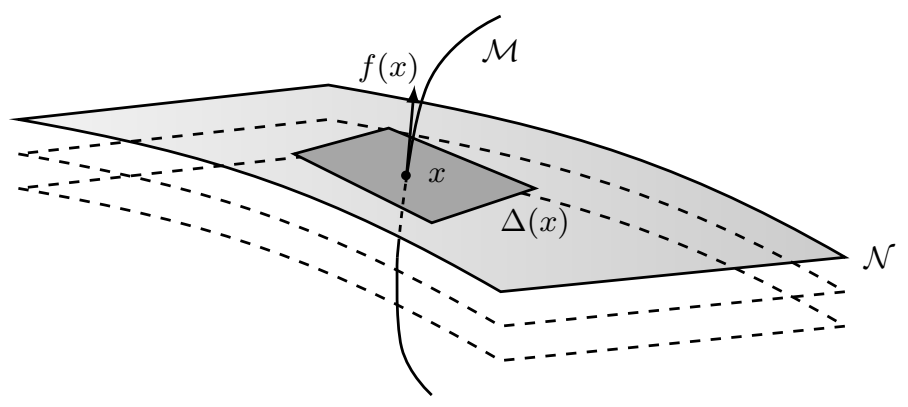

Fig. 1. Pictorial illustration of the notion of $f$-reducing manifold.

condition (ii) in Definition 3 is satisfied if and only if the (welldefined) restriction of the vector field $f$ to the manifold $\mathcal{M}$ coincides with the vector field $f_{2}$. With these premises, the manifold $\mathcal{M}$ is $f$-reducing if and only if the mapping $\pi$ solves the partial differential equation

$$
f_{1}\left(\pi\left(x_{2}\right), x_{2}\right)=\frac{\partial \pi}{\partial x_{2}} f_{2}\left(x_{2}\right) .
$$

We are now in the position to give the following definition.

Definition 4. Consider system (6), system (7) and the manifold $\mathcal{M}$ defined in 9p. The vector field $s: \mathbb{R}^{m} \rightarrow \mathbb{R}^{m}$ is said to be an eigenvalue of system (6) (with respect to $\mathcal{M}$ ) if $\mathcal{M}$ is the larges 5 $f$-reducing manifold such that the restriction of $f$ to $\mathcal{M}$ coincides with $s$.

Definition 4 is motivated by Lemma 2 and can be seen as a nonlinear counterpart of the notion of eigenvalue of a linear system. It formalizes the idea that an eigenvalue corresponds to the largest $f$-reducing manifold $\mathcal{M}$ and the (well-defined) restriction of system 6 to the manifold $\mathcal{M}$ is a copy of the dynamics of system (7). Similarly, the manifold $\mathcal{M}$ can be intepreted as the "generalized eigenmanifold" associated with the eigenvalue $s$.

Remark 2. Definition 4 implies that the vector field $f$ is an eigenvalue of system (6) (take $\mathcal{M}=\mathbb{R}^{n}$ and $\Delta=\{0\}$ ). For this reason, $f$ is referred to as a trivial eigenvalue, while all other eigenvalues are referred to as non-trivial.

For a linear system the existence of an eigenvalue corresponds to the solvability of a "reduced" Sylvester equation [7, p.21]. Similarly, every non-trivial eigenvalue of a nonlinear system implies the solvability of a "reduced" partial differential equation.

Theorem 3. Consider system (6). The vector field $s: \mathbb{R}^{m} \rightarrow \mathbb{R}^{m}$ is a non-trivial eigenvalue of system (6) if and only if, with respect to the decomposition $(10)$, there exists a mapping $\pi: \mathbb{R}^{m} \rightarrow \mathbb{R}^{n-m}$, locally defined in a neighborhood of the origin and such that $\pi(0)=0$, which solves the partial differential equation

$$
f_{1}\left(\pi\left(x_{2}\right), x_{2}\right)=\frac{\partial \pi}{\partial x_{2}} s\left(x_{2}\right) .
$$

To illustrate the ideas introduced above, we consider a simple example first studied in [29, p.14].

Example 1. Consider the planar system

$$
\dot{x}_{1}=-x_{1}+x_{2}^{2}, \quad \dot{x}_{2}=x_{2}, \quad x=\operatorname{col}\left(x_{1}, x_{2}\right) \in \mathbb{R}^{2} .
$$

The system possesses a unique hyperbolic equilibrium point at the origin. The (global) unstable manifold passing through the origin of system 13 is $\mathcal{M}=\left\{x \in \mathbb{R}^{2}: x_{1}=x_{2}^{2} / 3\right\}$. We show that $\mathcal{M}$ is an $f$-reducing manifold. Let $\Delta=\operatorname{span}\left\{e_{1}\right\}$ and note

\footnotetext{
${ }^{5}$ Largest is used locally in this context to mean that $\mathcal{M}$ locally contains any other $f$-reducing manifold. See also [10, p.294].
}

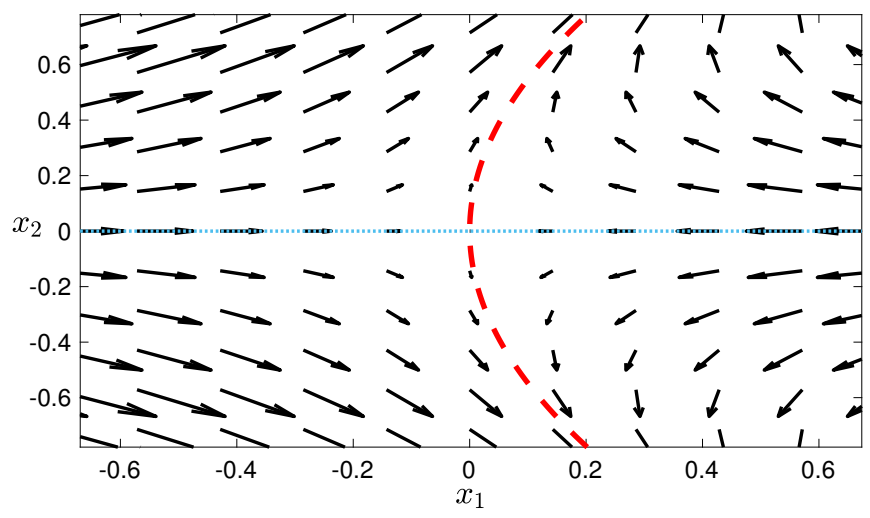

Fig. 2. Phase portrait of system (13), with the stable manifold $\mathcal{N}$ (dotted) and the unstable manifold $\mathcal{M}$ (dashed).

that $\Delta$ is non-singular, involutive and $f$-invariant. Direct inspection shows that the integral submanifolds of the distribution $\Delta$ are lines that are parallel to the $x_{1}$ axis. In particular, the maximal integral submanifold $\mathcal{N}$ of $\Delta$ passing through the origin coincides with the (global) stable manifold passing through the origin, i.e. $\mathcal{N}=\{x \in$ $\left.\mathbb{R}^{2}: x_{2}=0\right\}$. Fig. 2 displays the phase portrait of system 13 , with the stable manifold $\mathcal{N}$ (dotted) and the unstable manifold $\mathcal{M}$ (dashed). The tangent spaces of the manifolds $\mathcal{M}$ and $\mathcal{N}$ at the origin are $T_{0} \mathcal{M}=\operatorname{span}\left\{e_{2}\right\}$ and $T_{0} \mathcal{N}=\operatorname{span}\left\{e_{1}\right\}$, respectively. In accordance with the stable manifold theorem [29, p.14], these are complementary subspaces of the tangent space of the ambient manifold. Note also that the manifold $\mathcal{M}$ can be described as the graph of the mapping $\pi: \mathbb{R} \rightarrow \mathbb{R}$, defined as $\pi(\omega)=\frac{\omega^{2}}{3}$ for every $\omega \in \mathbb{R}$, which can be found eliminating time in 13 and solving the ordinary differential equation $\frac{d x_{1}}{d x_{2}}=-\frac{x_{1}}{x_{2}}+x_{2}$. Thus the restriction of system (13) to $\mathcal{M}$ is immersed into system (13), as the partial differential equation $-\pi+\omega^{2}=\frac{\partial \pi}{\partial \omega} \omega$ has a unique solution. This shows that $\mathcal{M}$ is an $f$-reducing manifold. We conclude that $\mathcal{M}$ is the largest $f$-reducing manifold with these properties. By Definition 4 this implies that the vector field $s: \mathbb{R} \rightarrow \mathbb{R}$, defined as $s(\omega)=\omega$ for every $\omega \in \mathbb{R}$, is a non-trivial eigenvalue of system 13 .

Example 1 raises a natural question: is the vector field $f_{1}: \mathbb{R} \rightarrow \mathbb{R}$ defined as $f_{1}(\omega)=-\omega$ for every $\omega \in \mathbb{R}$, a non-trivial eigenvalue of system (13)? The answer is affirmative and this can be verified as follows. With the aid of a symbolic software package (such as Maple $\left.{ }^{(}\right)$or MuPAD $\left.{ }^{\circledR}\right)$, the partial differential equation

$$
\frac{\partial \phi_{1}}{\partial x_{1}}\left(-x_{1}+x_{2}^{2}\right)+\frac{\partial \phi_{1}}{\partial x_{2}} x_{2}=-\phi_{1}
$$

can be seen to admit a solution $\phi_{1}: \mathbb{R}^{2} \rightarrow \mathbb{R}$ such that $\frac{\partial \phi_{1}}{\partial x_{1}}$ is not identically zero. This implies that the function $\phi: \mathbb{R}^{2} \rightarrow \mathbb{R}^{2}$, defined as $\phi(x)=\operatorname{col}\left(\phi_{1}(x), x_{2}\right)$ for every $x \in \mathbb{R}^{2}$, qualifies as a (local) change of coordinates around the origin. In these coordinates, system (13) is described by the equations $\dot{\xi}_{1}=-\xi_{1}, \dot{\xi}_{2}=\xi_{2}$, with $\xi=\operatorname{col}\left(\xi_{1}, \xi_{2}\right) \in \mathbb{R}^{2}$, from which one infers that the vector field $f_{1}$ is a non-trivial eigenvalue of the system.

The argument above can be generalized to show that a system described by equations of the form

$$
\dot{x}_{1}=f_{1}\left(x_{1}\right)+\varrho_{1}\left(x_{1}, x_{2}\right), \quad \dot{x}_{2}=f_{2}\left(x_{2}\right),
$$

in which $x=\operatorname{col}\left(x_{1}, x_{2}\right)$, with $x_{1} \in \mathbb{R}^{n-m}$ and $x_{2} \in \mathbb{R}^{m}$, and $f_{2}$ is a non-trivial eigenvalue, also possesses the non-trivial eigenvalue $f_{1}$ provided that the partial differential equation

$$
\frac{\partial \phi_{1}}{\partial x_{1}}\left(f_{1}+\varrho_{1}\right)+\frac{\partial \phi_{1}}{\partial x_{2}} f_{2}=f_{1} \circ \phi_{1},
$$


admits a "full rank" solution, as detailed in the following statement. Theorem 4. Consider system (15). Assume $f_{2}$ is a non-trivial eigenvalue of system (15) and the partial differential equation 16 admits a solution $\phi_{1}$ such that the matrix $\frac{\partial \phi_{1}}{\partial x_{1}}$ is full rank in a neighborhood of the origin. Then $f_{1}$ is a non-trivial eigenvalue of system 15 .

Proof. Under the stated assumptions the mapping $\phi: \mathbb{R}^{n} \rightarrow \mathbb{R}^{n}$, defined as $\phi(x)=\operatorname{col}\left(\phi_{1}(x), x_{2}\right)$ for every $x \in \mathbb{R}^{n}$, qualifies as a (local) change of coordinates around the origin. In the new coordinates system (15) is described by the equations $\dot{\xi}_{1}=f_{1}\left(\xi_{1}\right), \dot{\xi}_{2}=f_{2}\left(\xi_{2}\right)$, which shows that $f_{1}$ is a non-trivial eigenvalue of the system.

Remark 3. The existence of multiple non-trivial eigenvalues in a system can be also characterized in geometric terms using simultaneously integrable distributions [30]. For example, the existence of non-singular, involutive, simultaneously integrable distributions $\Delta_{\mathcal{M}}$ and $\Delta_{\mathcal{N}}$ such that $\operatorname{dim} \Delta_{\mathcal{M}}+\operatorname{dim} \Delta_{\mathcal{N}}=n$ and such that $\left[\theta_{1}, \theta_{2}\right]=0$ for every $\theta_{1} \in \Delta_{\mathcal{M}}$ and $\theta_{2} \in \Delta_{\mathcal{N}}$ implies the existence of a "full rank" solution of (16). By Theorem 4, this implies that if $f_{2}$ is a non-trivial eigenvalue of system 15 then so is $f_{1}$.

Example 2. The Kermack-McKendrick model for the spreading of an infectious disease is described by the equations [31]

$$
\dot{S}=-\varrho S I, \quad \dot{I}=\varrho S I-a I, \quad \dot{R}=a I,
$$

in which $S(t) \in \mathbb{R}_{+}$is the number of individuals susceptible to infection, $I(t) \in \mathbb{R}_{+}$is the number of infected individuals able to transmit the disease to other individuals, $R(t) \in \mathbb{R}_{+}$is the number of infected individuals who have successfully recovered, $\varrho \in \mathbb{R}_{+}$is the infection rate and $a \in \mathbb{R}_{+}$is the removal rate of infectives. Note that the number of individuals is constant over time, since $\dot{S}+\dot{I}+\dot{R}=0$. The number $R$ of infected individuals who have fully recovered can be studied setting $x_{1}=R, x_{21}=S, x_{22}=I$ and rewriting (17) as

$$
\dot{x}_{1}=a x_{22}, \dot{x}_{21}=-\varrho x_{21} x_{22}, \dot{x}_{22}=\varrho x_{21} x_{22}-a x_{22} .
$$

We show that the vector field $s: \mathbb{R}^{2} \rightarrow \mathbb{R}^{2}$, defined as

$$
s\left(x_{2}\right)=\left[\begin{array}{ll}
-\varrho x_{21} x_{22} & \varrho x_{21} x_{22}-a x_{22}
\end{array}\right]^{\prime}
$$

for every $x_{2}=\operatorname{col}\left(x_{21}, x_{22}\right) \in \mathbb{R}^{2}$, is an eigenvalue of system 18 . Note that system (18) admits an obvious cascade decomposition. As a result, we only need to verify that the partial differential equation

$$
a x_{22}=\frac{\partial \pi}{\partial x_{2}} s\left(x_{2}\right)
$$

admits a solution. Recall that the total number of individuals $N_{0} \in \mathbb{Z}_{+}$is constant over time, i.e. $x_{1}+x_{21}+x_{22}=N_{0}$. The triangle $\mathcal{T}$ described by the equations $x_{1}=N_{0}-x_{21}-x_{22}$, $0 \leq x_{21} \leq N_{0}, 0 \leq x_{22} \leq N_{0}$, is therefore an invariant manifold for system (18). This implies that the mapping $\pi: \mathbb{R}^{2} \rightarrow \mathbb{R}$, defined as $\pi\left(x_{2}\right)=N_{0}-x_{21}-x_{22}$ for every $x_{2} \in \mathbb{R}^{2}$, solves 20). This proves that $s$ is an eigenvalue of system (18).

\section{B. Poles and invariance notions}

We now provide a nonlinear enhancement of the notion of pole of a linear system. To this end, the notions of cascade decomposition and of $f$-reducing distribution are introduced.

Definition 5. System (6) is said to admit a cascade decomposition if there exist (local) coordinates $x=\operatorname{col}\left(x_{1}, x_{2}\right)$, with $x_{1} \in \mathbb{R}^{n-m}$ and $x_{2} \in \mathbb{R}^{m}$, in which system $[6$ is described by the equations

$$
\dot{x}_{1}=f_{1}\left(x_{1}, x_{2}\right), \dot{x}_{2}=f_{2}\left(x_{2}\right)+g_{2}\left(x_{2}\right) u, y=h_{1}\left(x_{1}\right),
$$

where $f_{1}: \mathbb{R}^{n} \rightarrow \mathbb{R}^{n-m}, f_{2}: \mathbb{R}^{m} \rightarrow \mathbb{R}^{m}, g_{2}: \mathbb{R}^{m} \rightarrow \mathbb{R}^{m}, h_{1}:$ $\mathbb{R}^{n-m} \rightarrow \mathbb{R}$ are such that $f_{1}(0,0)=0, f_{2}(0)=0, h_{1}(0)=0$, respectively.

Definition 6. Consider system [6. A non-singular, involutive, distribution $\Delta_{\mathcal{M}}$ locally defined around the origin is said to be $f$-reducing if its maximal integral submanifold $\mathcal{M}$ is $f$-reducing. The pair of distributions $\Delta_{\mathcal{M}}$ and $\Delta_{\mathcal{N}}$ are said to be $f$-reducing if $\Delta_{\mathcal{M}}$ and $\Delta_{\mathcal{N}}$ are $f$-reducing and complementary in a neighborhood $U$ of the origin, i.e. such that $\Delta_{\mathcal{M}}(x) \oplus \Delta_{\mathcal{N}}(x)=T_{x} \mathbb{R}^{n}$ for every $x \in U$.

Definition 6 formalizes the idea that if an $f$-reducing manifold $\mathcal{M}$ is the maximal integral submanifold of a distribution $\Delta_{\mathcal{M}}$ then the distribution should be called $f$-reducing too. Similarly, a pair of distributions $\Delta_{\mathcal{M}}$ and $\Delta_{\mathcal{N}}$ is referred to as $f$-reducing if $\Delta_{\mathcal{M}}$ and $\Delta_{\mathcal{N}}$ are both $f$-reducing and (locally) complementary, in analogy with the linear case.

We now provide conditions for a nonlinear system to admit a cascade decomposition. As a direct generalization of the assumptions of the linear case [23, Theorem 2], this requires the existence of two non-singular, involutive, complementary distributions $\Delta_{\mathcal{M}}$ and $\Delta_{\mathcal{N}}$ defined around the origin for which the following assumptions hold.

Assumption 1. There exist mappings $\theta: \mathbb{R}^{n} \rightarrow \mathbb{R}^{n}$ and $\gamma: \mathbb{R}^{n} \rightarrow \mathbb{R}$ such that the following conditions hold.

(i) $\left[f, \Delta_{\mathcal{N}}\right] \subset \Delta_{\mathcal{N}}$ and $\left[\theta, \Delta_{\mathcal{N}}\right] \subset \Delta_{\mathcal{N}}$

(ii) $g(x)$ is the projection of $\theta(x)$ onto $\Delta_{\mathcal{M}}(x)$.

(iii) $d h(x)$ is the projection of $d \gamma(x)$ onto $\Delta_{\mathcal{M}}^{\perp}(x)$.

Assumption 2. There exist mappings $\gamma_{1}: \mathbb{R}^{n-m} \rightarrow \mathbb{R}$ and $\gamma_{2}: \mathbb{R}^{m} \rightarrow \mathbb{R}$ such that the following conditions hold.

(iv) $\left[\mathrm{pr}_{\Delta_{\mathcal{N}}} \theta, \mathrm{pr}_{\Delta_{\mathcal{M}}} \theta\right]=0$.

(v) $\gamma(x)=\gamma_{1}\left(x_{1}\right)+\gamma_{2}\left(x_{2}\right)$.

(vi) $\Delta_{\mathcal{M}}$ is $\left(f-\theta \gamma_{2}\right)$-invariant.

Theorem 5. Consider system (6) and suppose Assumption 1 holds. Then system (6) admits a cascade decomposition of the form (21). Moreover, if Assumption 2 also holds then system (6) admits a cascade decomposition of the form

$$
\begin{aligned}
\dot{x}_{1} & =f_{1}\left(x_{1}\right)+g_{1}\left(x_{1}\right) h_{2}\left(x_{2}\right), \\
\dot{x}_{2} & =f_{2}\left(x_{2}\right)+g_{2}\left(x_{2}\right) u, \\
y & =h_{1}\left(x_{1}\right) .
\end{aligned}
$$

Proof. By condition (i) of Assumption 11, the distribution $\Delta_{\mathcal{N}}$ is invariant under the dynamic ${ }^{7}$ of the system $\dot{\chi}=f(\chi)+\theta(\chi) u$, with $\chi(t) \in \mathbb{R}^{n}$ and $u(t) \in \mathbb{R}$. This implies that there exist (local) coordinates $x=\operatorname{col}\left(x_{1}, x_{2}\right)$, with $x_{1} \in \mathbb{R}^{n-m}, x_{2} \in \mathbb{R}^{m}$ and $n-m$ the dimension of $\Delta_{\mathcal{N}}$ in a neighborhood of the origin, in which the vector fields $f$ and $\theta$ can be written as

$$
f(x)=\left[\begin{array}{c}
f_{1}\left(x_{1}, x_{2}\right) \\
f_{2}\left(x_{2}\right)
\end{array}\right] \quad \theta(x)=\left[\begin{array}{c}
\theta_{1}\left(x_{1}, x_{2}\right) \\
\theta_{2}\left(x_{2}\right)
\end{array}\right] .
$$

Condition (ii) of Assumption 1 implies that, in the above coordinates, the vector field $g$ can be written as $g(x)=\left[\begin{array}{ll}0 & \theta_{2}\left(x_{2}\right)^{\prime}\end{array}\right]^{\prime}$. On the other hand, by condition (iii) of Assumption 1$] d h=\left[\begin{array}{ll}d \gamma_{1} & 0\end{array}\right]$. This implies $\frac{\partial h}{\partial x_{2}}=0$ and hence $h(x)=\gamma\left(x_{1}\right)$. This proves the first claim.

A direct computation gives

$$
\left[\mathrm{pr}_{\Delta_{\mathcal{N}}} \theta, \mathrm{pr}_{\Delta_{\mathcal{M}}} \theta\right]=\left[\begin{array}{c}
\frac{\partial \theta_{1}}{\partial x_{2}} \theta_{2} \\
0
\end{array}\right] .
$$

By condition (iv) of Assumption 2 and by the minimality of system (6), this implies $\frac{\partial \theta_{1}}{\partial x_{2}}=0$. Thus, there exist $\tilde{\theta}_{1}: \mathbb{R}^{m} \rightarrow \mathbb{R}^{m}$ such that

${ }^{6} \mathrm{pr}_{\Delta} \theta$ denotes the projection of the vector field $\theta$ onto the distribution $\Delta$.

${ }^{7} \mathrm{~A}$ distribution $\Delta$ is invariant under the dynamics of the system $\dot{x}=f(x)+g(x) u$, with $x(t) \in \mathbb{R}^{n}$ and $u(t) \in \mathbb{R}$, if $[f, \Delta] \subset \Delta$ and $[g, \Delta] \subset \Delta[32, \mathrm{p} .333]$. 
$\theta_{1}(x)=\tilde{\theta}_{1}\left(x_{1}\right)$ for every $x=\operatorname{col}\left(x_{1}, x_{2}\right) \in \mathbb{R}^{n}$ locally around the origin. By conditions (v) and (vi) of Assumption 2, the distribution $\Delta_{\mathcal{M}}$ is $\left(f-\theta \gamma_{2}\right)$-invariant and hence

$$
f(x)-\theta(x) \gamma(x)=\left[\begin{array}{c}
f_{1}\left(x_{1}, x_{2}\right)-\tilde{\theta}_{1}\left(x_{1}\right) \gamma_{2}\left(x_{2}\right) \\
f_{2}\left(x_{2}\right)-\theta_{2}\left(x_{2}\right) \gamma_{2}\left(x_{2}\right)
\end{array}\right]
$$

gives $f_{1}\left(x_{1}, x_{2}\right)-\tilde{\theta}_{1}\left(x_{1}\right) \gamma\left(x_{2}\right)=\varphi_{1}\left(x_{1}\right)$. Putting things together yields

$$
f(x)=\left[\begin{array}{c}
\varphi_{1}\left(x_{1}\right)+\tilde{\theta}_{1}\left(x_{1}\right) \gamma_{2}\left(x_{2}\right) \\
f_{2}\left(x_{2}\right)
\end{array}\right], g(x)=\left[\begin{array}{c}
0 \\
\theta_{2}\left(x_{2}\right)
\end{array}\right],
$$

and $h(x)=\gamma_{1}\left(x_{1}\right)$, from which the second claim follows.

We are now ready to give the following definition.

Definition 7. Consider system (6), system (7) and the manifold $\mathcal{M}$ defined in 97. Let $\Delta_{\mathcal{M}}$ and $\Delta_{\mathcal{N}}$ be $f$-reducing distributions that satisfy Assumption 1 and let $\mathcal{M}$ be the maximal integral submanifold of $\Delta_{\mathcal{M}}$. The vector field $s: \mathbb{R}^{m} \rightarrow \mathbb{R}^{m}$ is said to be a pole of system (6) if $s$ is a non-trivial eigenvalue of system (6) (with respect to $\mathcal{M})$.

Definition 7 is motivated by Lemma 3 and can be seen as a coordinatefree version of the notion of pole introduced in [23]. Note that, as a direct consequence of Definition 7, if $s: \mathbb{R}^{m} \rightarrow \mathbb{R}^{m}$ is a pole of system (6) then the system admits a cascade decomposition. Note also that if the vector field $s: \mathbb{R}^{m} \rightarrow \mathbb{R}^{m}$ is a non-trivial eigenvalue of system (6) and $\Delta_{\mathcal{M}}$ and $\Delta_{\mathcal{N}}$ are corresponding $f$-reducing distributions then the distribution $\Delta_{\mathcal{N}}$ is, by definition, $f$-invariant. Thus the condition $\left[f, \Delta_{\mathcal{N}}\right] \subset \Delta_{\mathcal{N}}$ is always satisfied and $s$ is a pole of system (6) if and only if there exist a vector field $\theta: \mathbb{R}^{n} \rightarrow \mathbb{R}^{n}$ and a function $\gamma: \mathbb{R}^{n} \rightarrow \mathbb{R}$ such that (i) ${ }^{*}\left[\theta, \Delta_{\mathcal{N}}\right] \subset \Delta_{\mathcal{N}}$, (ii) ${ }^{*} g(x)$ is the projection of $\theta(x)$ onto $\Delta_{\mathcal{M}}(x)$, and (iii) ${ }^{*} d h(x)$ is the projection of $d \gamma(x)$ onto $\Delta_{\mathcal{M}}^{\perp}(x)$.

We now illustrate the notion of pole introduced in Definition 7 with a simple worked-out example.

Example 3. Consider the system

$$
\dot{x}_{1}=-x_{1}+x_{2}^{2}, \quad \dot{x}_{2}=x_{2}+u, \quad y=x_{1},
$$

in which $x(t) \in \mathbb{R}^{2}, u(t) \in \mathbb{R}, y(t) \in \mathbb{R}$. Note that system $(13)$ is the autonomous system associated with system 23 . Thus, in view of Example 1, the vector field $s: \mathbb{R} \rightarrow \mathbb{R}$, defined as $s(\omega)=\omega$ for every $\omega \in \mathbb{R}$, is a non-trivial eigenvalue of system [23.

The purpose of this example is to show that the vector field $s$ is a pole of system 23. To this end, consider the distributions $\Delta_{\mathcal{M}}=\operatorname{span}\left\{e_{2}\right\}$ and $\Delta_{\mathcal{N}}=\operatorname{span}\left\{e_{1}\right\}$ and note that these are $f$-reducing at the origin, by Example 1 Thus, we only need to show that Assumption 1 holds. Define $\theta: \mathbb{R}^{2} \rightarrow \mathbb{R}^{2}$ as $\theta(x)=e_{1}+e_{2}$ and $\gamma: \mathbb{R}^{n} \rightarrow \mathbb{R}$ as $\gamma(x)=x_{1}+x_{2}^{2}$ for every $x \in \mathbb{R}^{2}$, respectively. A direct computation gives $\left[f, e_{1}\right]=e_{1}$ and $\left[\theta, e_{1}\right]=0$. This implies that condition (i) of Assumption 1 is satisfied. On the other hand, conditions (ii) and (iii) of Assumption 1 are seen to hold by direct inspection. We conclude that $s$ is indeed a pole of system 23. Finally, we emphasize that Assumption 2 can be also seen to hold by direct computation and that, in agreement with Theorem 5 system 23 is described by equations of the form 22 .

The existence of multiple poles in a system can be characterized in geometric terms much in the same way as the existence of multiple non-trivial eigenvalues. For example, system (6) possesses two distinct poles if there exist simultaneously integrable, $f$-reducing distributions $\Delta_{\mathcal{M}}$ and $\Delta_{\mathcal{N}}$ such that system [6] can be written, with respect to the decompositions induced by these distributions, both as (22) and as

$$
\begin{aligned}
\dot{z}_{1} & =f_{1}\left(z_{1}\right)+\tilde{g}_{1}\left(z_{1}\right) u, \\
\dot{z}_{2} & =f_{2}\left(z_{2}\right)+\tilde{g}_{2}\left(z_{2}\right) \tilde{h}_{1}\left(z_{1}\right), \\
y & =\tilde{h}_{2}\left(z_{2}\right),
\end{aligned}
$$

respectively. We emphasize that this is not always possible, in general. For example, consider the system (23) and note that it is described by equations of the form 22. Moreover, on the basis of Example 3 the vector field $s: \mathbb{R} \rightarrow \mathbb{R}$, defined as $s(\omega)=\omega$ for every $\omega \in \mathbb{R}$, is a pole of system 23 . However, the vector field $\tau: \mathbb{R} \rightarrow \mathbb{R}$, defined as $\tau(\omega)=-\omega$ for every $\omega \in \mathbb{R}$, is not a pole of system 23, since system (23) cannot be described by equations of the form (24). This can be seen as follows. If the system could be described by equations of the form (24) then there would exist a (local) change of coordinates $z=\phi(x)$ and functions $\psi_{f}: \mathbb{R}^{2} \rightarrow \mathbb{R}$ and $\psi_{g}: \mathbb{R} \rightarrow \mathbb{R}$ such that

$$
\left[\frac{\partial \phi}{\partial x} f(x) \frac{\partial \phi}{\partial x} g(x)\right]_{x=\phi^{-1}(z)}=\left[\begin{array}{cc}
-z_{1} & \psi_{g}\left(z_{1}\right) \\
z_{2}+\psi_{f}\left(z_{1}, z_{2}\right) & 0
\end{array}\right],
$$

from which a contradiction immediately follows.

\section{Moments at a pole and steady-state impulse responses}

Definition 8. Consider system (6) and suppose 21) holds with respect to the (local) coordinates $x=\operatorname{col}\left(x_{1}, x_{2}\right)$, with $x_{1} \in \mathbb{R}^{n-m}$ and $x_{2} \in \mathbb{R}^{m}$. Let $s: \mathbb{R}^{m} \rightarrow \mathbb{R}^{m}$ be a pole of system (6) described in (local) coordinates by $f_{2}$. The moment of system (6) at $s$ is defined as the function $h_{1} \circ \pi$, with $\pi$ the unique solution of (11).

In analogy with the linear case, the moment at a pole uniquely determines the steady-state impulse response of the output of a nonlinear system, provided that some assumptions hold.

Theorem 6. Consider system (6) and suppose 21] holds with respect to the (local) coordinates $x=\operatorname{col}\left(x_{1}, x_{2}\right)$, with $x_{1} \in \mathbb{R}^{n-m}$ and $x_{2} \in \mathbb{R}^{m}$. Let $s: \mathbb{R}^{m} \rightarrow \mathbb{R}^{m}$ be a pole of system (6) described in (local) coordinates by $f_{2}$. Assume the equilibrium at the origin of the system $\dot{x}_{1}=f_{1}\left(x_{1}, 0\right)$ is locally exponentially stable, the system $\dot{x}_{2}=f_{2}\left(x_{2}\right)$ is Poisson stable and the pair $\left(f_{2}, g_{2}(0)\right)$ is exciting 8 Then the moment of system 6 at $s$ uniquely determines the steadystate impulse response of the output of system 6 .

Proof. To begin with note that the pole $s$ of system (6) is welldefined, since under the stated assumptions there exists a (well-defined) center manifold [34] described by the equation $x_{1}=\pi\left(x_{2}\right)$. Let $\varepsilon=x_{1}-\pi\left(x_{2}\right)$. Differentiating $\varepsilon$ with respect to time and using 11 yields $\dot{\varepsilon}=f_{1}\left(\varepsilon+\pi\left(x_{2}\right), x_{2}\right)-f_{1}\left(\pi\left(x_{2}\right), x_{2}\right)-L_{g_{2}} \pi\left(x_{2}\right) u$, which, owing to $u=\delta_{0}$ and the local exponential stability assumption, implies $\lim _{t \rightarrow \infty}\|\varepsilon(t)\|=0$. This shows that the (center) manifold $\mathcal{M}=\left\{x \in \mathbb{R}^{n}: x_{1}=\pi\left(x_{2}\right), x_{2} \in \mathbb{R}^{m}\right\}$, with $\pi$ the unique solution of (11), is an attractive invariant manifold of system (1). Thus the steady-state impulse response of system (1) is well-defined and the output of system (1) is $y(t)=h_{1}\left(\pi \circ x_{2}(t)+\varepsilon(t)\right)$, in which $\varepsilon$ is an exponentially decaying function. Thus, the steady-state impulse response of the output of system (6) is $y_{i r}(t)=h_{1} \circ \pi \circ x_{2}(t)$, for $t>0$. Hence, in view of Definition 8 the claim is proved.

Remark 4. The proof of Theorem 6 assumes implicitly that the input $u=\delta_{0}$ is such that the corresponding initial condition $x_{2}(0)$ belongs to the basin of attraction of the equilibrium at the origin. If that is not the case, it suffices to rescale the "amplitude" of the input. $\triangle$

Remark 5. Theorem 6 provides sufficient conditions for the moment of a system to uniquely determine the steady-state impulse response of

\footnotetext{
${ }^{8}$ The pair $\left(f, x_{0}\right)$ is said to be exciting if $\operatorname{dim} E\left(x_{0}\right)=n$, in which $E(x)=$ span $\left\{\theta_{0}(x), \theta_{1}(x), \theta_{2}(x), \ldots\right\}$, with $\theta_{k+1}(x)=\frac{\partial \theta_{k}}{\partial x} f(x)$ for $k \in \mathbb{Z}_{+}$and $\theta_{0}$ the identity map. The reader is referred to [33] for more detail.
} 
the system. However, this situation can be encountered under different circumstances and, in fact, different definitions of moment can be given. This issue is extensively discussed in [35].

Remark 6. If the relative degree of system (6) is not sufficiently large it is impossible to realize the system as a cascade of two strictly proper systems. In this case, however, the moments at a pole can be still characterized using a cascade of singular systems and combining the results of the present paper with those of [36].

\section{MOdEL REDUCTION BY MOMENT MATCHING AT POLES}

We now provide a nonlinear counterpart of the results established for linear systems in [22].

Definition 9. Consider system (6) and suppose (21) holds with respect to the (local) coordinates $x=\operatorname{col}\left(x_{1}, x_{2}\right)$, with $x_{1} \in \mathbb{R}^{n-m}$ and $x_{2} \in \mathbb{R}^{m}$. Let $s: \mathbb{R}^{m} \rightarrow \mathbb{R}^{m}$ be a pole of system (6) described in (local) coordinates by $f_{2}$. Assume the equilibrium at the origin of system $\dot{x}_{1}=f_{1}\left(x_{1}, 0\right)$ is locally exponentially stable, the system $\dot{x}_{2}=f_{2}\left(x_{2}\right)$ is Poisson stable and the pair $\left(f_{2}, g_{2}(0)\right)$ is exciting. The system

$$
\dot{\xi}=\phi(\xi)+\gamma(\xi) u, \quad \psi=\kappa(\xi)
$$

with $\xi(t) \in \mathbb{R}^{m}, u(t) \in \mathbb{R}, \psi(t) \in \mathbb{R}$, is said to be a reduced order model at $s$ of system (6) if there exists an invertible mapping $p: \mathbb{R}^{m} \rightarrow \mathbb{R}^{m}$, locally defined in a neighborhood of the origin, with $p(0)=0$, such that

$$
\frac{\partial p}{\partial \xi} \phi(\xi)=f_{2}(p(\xi)), \frac{\partial p}{\partial \xi} \gamma(\xi)=g_{2}(p(\xi)), \kappa(\xi)=h_{1}(\pi(p(\xi))),
$$

where $\pi$ is the unique solution of the partial differential equation (11)

Definition 9 is motivated by the following result.

Proposition 1. Consider system (6) and suppose 21) holds with respect to the (local) coordinates $x=\operatorname{col}\left(x_{1}, x_{2}\right)$, with $x_{1} \in \mathbb{R}^{n-m}$ and $x_{2} \in \mathbb{R}^{m}$. Let $s: \mathbb{R}^{m} \rightarrow \mathbb{R}^{m}$ be a pole of system (6) described in (local) coordinates by $f_{2}$. Suppose system 26 is a reduced order model of system (6) at $s$. Then system (26) matches the moment of system 6 at $s$.

Proof. By hypothesis, system 26 is a reduced order model of system (6) at $s$ and thus, by definition, there exists an invertible mapping $p: \mathbb{R}^{m} \rightarrow \mathbb{R}^{m}$, locally defined in a neighborhood of the origin, with $p(0)=0$, and 27) holds, where $\pi$ is the unique solution of the partial differential equation (11). This implies that $p$ qualifies as a (local) change of coordinates and, setting $\zeta=p(\xi)$, system 26) can be rewritten as $\dot{\zeta}=f_{2}(\zeta)+g_{2}(\zeta) u, \psi=h_{1} \circ \pi(\zeta)$. By Definition 7 this shows that the reduced order model 26] has a pole at $s$. Moreover, the steady-state response of the output of system (1) and of the reduced order model 26 with input $u=\delta_{0}$ coincide. This, in view of Theorem 6 proves the claim.

The model reduction framework and the coordinate-free characterization of eigenvalues and poles presented in this paper can be extended, e.g., to singular systems [37] using the notion of deflating subspace [38], a generalization of the notion of $A$-reducing subspace which has been employed for model reduction purposes in [39]. Extensions to other classes of systems, including, e.g., timedelay systems [40] and systems described by integro-differential equations [41], are also possible. A nonlinear counterpart of the notion of reduced order model achieving moment matching simultaneously at ordinary points and at poles can be also defined.
1) A family of reduced order models. Selecting $p(\xi)=\xi$ in 27) yields a reduced order model of system (1) at $s$ described by equations of the form [26, with $\phi(\xi)=f_{2}(\xi), \gamma(\xi)=g_{2}(\xi)$, $\kappa(\xi)=h_{1} \circ \pi(\xi)$. Observe that the reduced order model obtained has a pole at $s$ and that the reduced order models achieves moment matching at $s$. Pushing the analogy with the linear case, the approach described above can be considered as a nonlinear counterpart of that of modal approximation [25, Chapter 9]. The following example illustrates the proposed model reduction procedure.

Example 4. Consider the system

$$
\dot{x}_{1}=-x_{1}-x_{1} x_{3}, \dot{x}_{2}=\Omega x_{3}, \dot{x}_{3}=-\Omega x_{2}+u, y=x_{1},
$$

in which $x(t) \in \mathbb{R}^{3}, u(t) \in \mathbb{R}, y(t) \in \mathbb{R}$ and $\Omega \in \mathbb{R}_{+}$. The system possesses a unique equilibrium point at the origin and an inherent cascade decomposition. Moreover, the partial differential equation

$$
-\pi\left(1+\omega_{2}\right)=\Omega \omega_{2} \frac{\partial \pi}{\partial \omega_{1}}-\Omega \omega_{1} \frac{\partial \pi}{\partial \omega_{2}}
$$

admits the closed form solution

$$
\pi(\omega)=\left(\omega_{1}^{2}+\omega_{2}^{2}\right) e^{-\frac{1}{\Omega}\left(\omega_{1}+\arctan \left(\frac{\omega_{1}}{\omega_{2}}\right)\right)} .
$$

Observe that the function $\pi$ is continuous on the set $\mathcal{S}=\left\{\omega \in \mathbb{R}^{2}\right.$ : $\left.\omega_{2}=0\right\}$. Note, however, that $\mathcal{S}$ is not an invariant set of system 28. and that setting $u=\delta_{0}$ yields $x_{3}(0)=1$. This allows concluding that the vector field $s: \mathbb{R}^{2} \rightarrow \mathbb{R}^{2}$, defined as $s(\omega)=\left[\begin{array}{ll}\Omega \omega_{2} & -\Omega \omega_{1}\end{array}\right]^{\prime}$, for every $\omega \in \mathbb{R}^{2}$, is a pole of system 28. Note that system [28. satisfies the assumptions of Theorem 6 The moment of the system at $s$ is

$$
h_{1} \circ \pi(\omega)=\left(\omega_{1}^{2}+\omega_{2}^{2}\right) e^{-\frac{1}{\Omega}\left(\omega_{1}+\arctan \left(\frac{\omega_{1}}{\omega_{2}}\right)\right)}
$$

and a reduced order model achieving moment matching at $s$ is

$\dot{\xi}_{1}=\Omega \xi_{2}, \dot{\xi}_{2}=-\Omega \xi_{1}+u, \psi=\left(\xi_{1}^{2}+\xi_{2}^{2}\right) e^{-\frac{1}{\Omega}\left(\xi_{1}+\arctan \left(\frac{\xi_{1}}{\xi_{2}}\right)\right)}$.

Note that the steady-state impulse response of the reduced order model [32] is well-defined, since along the solutions of the reduced order model 32 one has $\arctan \left(\frac{\xi_{1}}{\xi_{2}}\right)=\Omega t$ for every $t \in \mathbb{R}_{+}$. Simulations have been run using an explicit Runge-Kutta (2,3)-order integration method and selecting the parameter $\Omega=5$. The initial conditions of system (28) and of the reduced order model (32) have been selected as $x(0)=\left[\begin{array}{lll}1 & 0 & 0\end{array}\right]^{\prime}$ and as $\xi(0)=\left[\begin{array}{ll}0 & 0\end{array}\right]^{\prime}$, respectively. Figure 3 displays the output of system (28) and of the reduced order model (32) with input $u=\delta_{0}$ (top) and the absolute value of the approximation error (bottom). Note that the time histories of the output of system (28) and of the reduced order model (32) are almost overlapped and that the approximation error converges to zero exponentially.

\section{A. Forced oscillations and reduced order models}

The analysis of forced oscillations in a system is a classical topic in systems theory $[29,42]$. The purpose of this section is to show that, borrowing ideas from [1], this phenomenon can be approximated with arbitrary accuracy and without any assumption on the solution of the system (such as periodicity) by computing an appropriate reduced order model.

Consider system (6) and assume its input is generated by the steady-state impulse response of a system of the form

$$
\dot{\omega}=S \omega+M v, \quad \varsigma=L \omega,
$$

in which $\omega(t) \in \mathbb{R}^{m}, \quad v(t) \in \mathbb{R}, \quad \varsigma(t) \in \mathbb{R} \quad$ and $\quad S \in \mathbb{R}^{m \times m}$, $M \in \mathbb{R}^{m}, L \in \mathbb{R}^{1 \times m}$ are constant matrices such that $S+S^{\prime}=0$ 

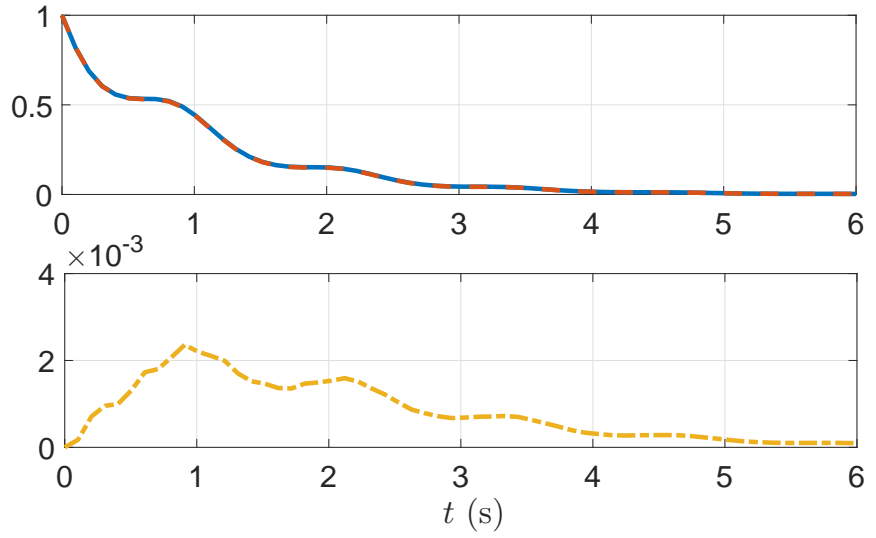

Fig. 3. Top graph: time histories of the output of system (28) (solid) and of the reduced order model [32] (dashed) with input $\boldsymbol{u}=\boldsymbol{\delta}_{\mathbf{0}}$. Bottom graph: time histories of the absolute value of the approximation error (dash-dotted).

and $(S, M, L)$ is minimal. The input mode 9 allows describing a wide range of oscillatory signals and is inspired and motivated by the exosystem description [10]. The presence of forced oscillations in system (6) is modeled considering the interconnection of systems [6] and 33 , with $u=\varsigma$, which reads as

$$
\dot{x}=f(x)+g(x) L \omega, \quad \dot{\omega}=S \omega+M v, \quad y=h(x) .
$$

Setting $x_{1}=x$ and $x_{2}=\omega$, system (34) is immediately seen to be of the form 21. Assuming that the equilibrium at the origin of system (6) is locally exponentially stable, the theory of the steadystate response of a nonlinear system [10] guarantees that there exists a (unique) solution of the partial differential equation

$$
f(\pi(\omega))+g(\pi(\omega)) L \omega=\frac{\partial \pi}{\partial \omega} S \omega
$$

and that the equation $x=\pi(\omega)$ describes a center manifold for system (34). This implies that the vector field $s: \mathbb{R}^{m} \rightarrow \mathbb{R}^{m}$, defined as $s(\omega)=S \omega$ for every $\omega \in \mathbb{R}^{m}$, is a pole of system 34. Moreover, the (well-defined) moment of system (34) at $s$ can be described in terms of the steady-state impulse response of the output of system (6), since the hypotheses of Theorem 6 are satisfied.

A natural reduced order model of system 34 at $s$ is described by the equations

$$
\dot{\xi}=S \xi+M u, \quad \psi=\operatorname{Lh}(\pi(\xi)),
$$

in which $\pi$ is the unique solution of the partial differential equation (35). Note that, in general, it is not possible to compute a closed form expression of the solution of 35 and, hence, of the reduced order model (36). However, the solution of 35 can be approximated up to arbitrary precision using a series expansion as follows. Formally, let $\pi(\omega)=\sum_{i=0}^{\infty} \pi_{i}(\omega)$, with $\pi_{i}(\omega)=\operatorname{col}\left(\pi_{i}^{1}(\omega), \pi_{i}^{2}(\omega), \cdots, \pi_{i}^{m}(\omega)\right)$ and $\pi_{i}^{j}(\omega)$ a homogeneous polynomial of degree $i$ in $\omega$ with unknown coefficients. Then substitute the power series expansion of $\pi$ into 35 and equate the coefficients of both sides to determine the coefficients of $\pi$ up to the desired degree of accuracy. We illustrate the ideas described above by means of a simple worked-out example.

Example 5. The forced Duffing oscillator is described by the equation [29]

$$
\ddot{\chi}+\delta \dot{\chi}+\alpha \chi+\beta \chi^{3}=\gamma \cos (\Omega t)
$$

${ }^{9}$ See also [43], where a similar model has been used to provide a Lyapunov characterization of forced oscillations in a system.
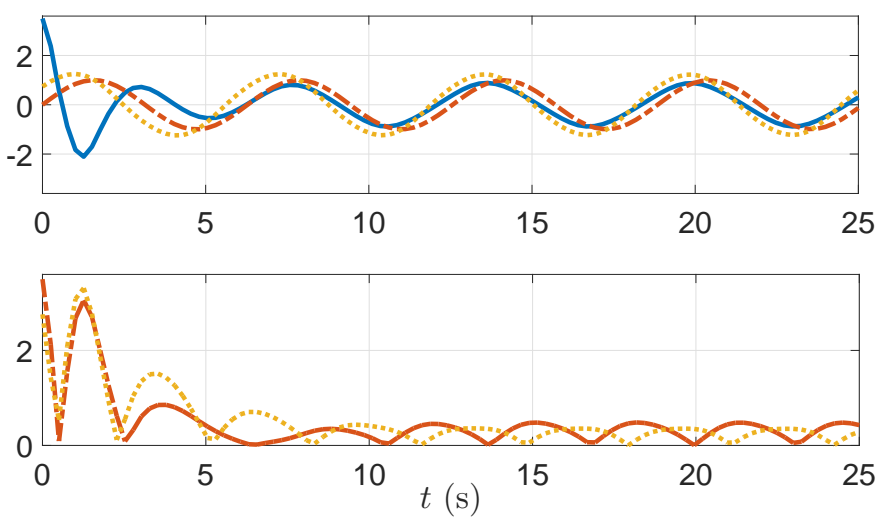

Fig. 4. Top graph: time histories of the output of system $(38)$ and of the approximate reduced order model 44 with input $\boldsymbol{u}=\boldsymbol{\delta}_{\mathbf{0}}: \boldsymbol{y}$ (solid), $\psi^{[1]}$ (dash-dotted) and $\psi^{[3]}$ (dotted). Bottom graph: time histories of the absolute value of the approximation errors $\boldsymbol{y}-\boldsymbol{\psi}^{[1]}$ (dash-dotted) and $\boldsymbol{y}-\boldsymbol{\psi}^{[3]}$ (dotted).

with $\chi(t) \in \mathbb{R}$ and $\alpha, \beta, \gamma, \delta, \Omega \in \mathbb{R}_{+}$, and can be modeled by the state-space system

$$
\begin{gathered}
\dot{x}_{1}=x_{2}, \quad \dot{x}_{2}=-\alpha x_{1}-\beta x_{1}^{3}-\delta x_{2}+\gamma x_{4}, \\
\dot{x}_{3}=\Omega x_{4}, \dot{x}_{4}=-\Omega x_{3}+u, \quad y=x_{1} .
\end{gathered}
$$

in which $x(t) \in \mathbb{R}^{4}, u(t) \in \mathbb{R}, y(t) \in \mathbb{R}$. Note that every solution of 37 can be computed as the response of the output of system 38. with input $u=\delta_{0}$ and an appropriate initial condition. The system possesses an equilibrium point at the origin and has an inherent cascade decomposition. Moreover, the equilibrium at the origin of the (unforced) Duffing equation (37), with $\gamma=0$, is globally exponentially stable. Thus, there exists a (unique) solution of the system of partial differential equation

$$
\begin{aligned}
\pi_{2} & =\Omega \omega_{2} \frac{\partial \pi_{1}}{\partial \omega_{1}}-\Omega \omega_{1} \frac{\partial \pi_{1}}{\partial \omega_{2}}, \\
-\alpha \pi_{1}-\beta \pi_{1}^{3}-\delta \pi_{2}+\gamma \omega_{2} & =\Omega \omega_{2} \frac{\partial \pi_{2}}{\partial \omega_{1}}-\Omega \omega_{1} \frac{\partial \pi_{2}}{\partial \omega_{2}} .
\end{aligned}
$$

This means that the vector field $s: \mathbb{R}^{2} \rightarrow \mathbb{R}^{2}$, defined as $s(\omega)=$ $\left[\Omega \omega_{2}-\Omega \omega_{1}\right]^{\prime}$, for every $\omega \in \mathbb{R}^{2}$, is a pole of system [38. Note that system 38 satisfies the assumptions of Theorem 6 A reduced order model of system 38 achieving moment matching at $s$ is

$$
\dot{\xi}_{1}=\Omega \xi_{2}, \quad \dot{\xi}_{2}=-\Omega \xi_{1}+u, \quad \psi=\pi\left(\xi_{2}\right),
$$

in which $\pi$ is the unique solution of 39. Note that it is not possible to compute the solution of 39] explicitly. However, an approximate reduced order model of system (38) at $s$ may be defined by the equations

$$
\dot{\xi}_{1}=\Omega \xi_{2}, \quad \dot{\xi}_{2}=-\Omega \xi_{1}+u, \quad \psi^{[j]}=\sum_{i=0}^{j} \pi_{i}\left(\xi_{2}\right),
$$

with $\psi^{[j]}$ the Taylor approximation of $\pi$ of order $j$ about the origin.

Simulations have been run to assess the properties of the approximate reduced order model 41. System (38) and the approximate reduced order model (41) have been numerically integrated using an explicit Runge-Kutta (2,3)-order integration method and selecting the parameters $\alpha=1, \beta=1, \gamma=1, \delta=1, \Omega=5$. The initial conditions of system (38) and of the approximate reduced order model 41] have been selected as $x(0)=\left[\begin{array}{llll}3.5 & 0 & 0 & 0\end{array}\right]^{\prime}$ and as $\xi(0)=\left[\begin{array}{ll}0 & 0\end{array}\right]^{\prime}$, respectively. Figure 4 (top) displays the steadystate impulse output response of system (28) and of the reduced order model 41] obtained by truncating the Taylor series expansion 
defining $\psi$ to the first and third order, respectively. Figure 4 (bottom) displays the absolute value of the approximation errors $y-\psi^{[1]}$ and $y-\psi^{[3]}$, respectively. Note that, in steady-state, $\max \left|y-\psi^{[1]}\right|=0.4287>\max \left|y-\psi^{[3]}\right|=0.2874$ and that the approximation error decreases by adding terms in the Taylor series expansion defining the output of the approximate reduced order model.

Remark 7. There exist several methods and tools to approximate the behavior of forced oscillations in a system, including the harmonic balance method [42], perturbation methods [42], the homotopy analysis [44]. In contrast with these methods, the use of (approximate) reduced order models does not require any assumption on the structure of the solution of the system. Moreover, the behavior of forced oscillations in a system can be determined up to arbitrary precision analogous to the use of Fourier series expansions, which, however, requires the knowledge of the period of the solution (which may not even be periodic). One limitation of our method, on the other hand, is that it can be storage expensive for large-scale systems.

\section{CONCLUSION}

Geometric control theory has been used to define a nonlinear enhancement of the notions of eigenvalue, pole and moment at a pole. These notions have been linked to solutions of "reduced" partial differential equations, cascade decompositions and steady-state impulse responses. The theory developed has proved instrumental in posing and solving the model reduction problem by moment matching at poles for nonlinear systems. Families of reduced order models achieving moment matching at a pole have been obtained, thus providing a nonlinear counterpart of modal approximation.

\section{REFERENCES}

[1] A. Astolfi, "Model reduction by moment matching for linear and nonlinear systems," IEEE Trans. Autom. Control, vol. 55, no. 10, pp. 2321-2336, 2010 .

[2] K. Ogata, Modern control engineering (5th edition). Englewood Cliffs, NJ, USA: Prentice-Hall, 2010.

[3] A. Padoan and A. Astolfi, "Model reduction by moment matching at isolated singularities for linear systems: a complex analytic approach," in Proc. 20th IFAC World Congr., Toulouse, France, 2017, pp. 6524-6528.

[4] W. Kaplan, Operational methods for linear systems. Reading, MA USA: Addison-Wesley, 1962.

[5] L. A. Zadeh and C. A. Desoer, Linear system theory: the state space approach. New York, NY, USA: McGraw-Hill, 1963.

[6] R. W. Brockett, Finite dimensional linear systems. New York, NY, USA: Wiley, 1970

[7] W. M. Wonham, Linear multivariable control: a geometric approach (2nd edition). New York, NY, USA: Springer-Verlag, 1979.

[8] T. Kailath, Linear systems. Englewood Cliffs, NJ, USA: Prentice-Hall, 1980.

[9] E. D. Sontag, Mathematical control theory: deterministic finite dimensional systems (2nd edition). New York: Springer-Verlag, 1998.

[10] A. Isidori, Nonlinear control systems (3rd edition). New York, NY, USA: Springer-Verlag, 1995.

[11] H. Nijmeijer and A. Van der Schaft, Nonlinear dynamical control systems. New York, NY, USA: Springer-Verlag, 1990.

[12] A. M. Bloch, Nonholonomic mechanics and control. New York, NY, USA: Springer-Verlag, 2003.

[13] A. M. Perdon, C. H. Moog, and G. Conte, "The pole-zero structure of nonlinear control systems," in 7th IFAC Symp. Nonlinear Control Syst., Pretoria, South Africa, 2007, pp. 717-720.

[14] M. Halás and C. H. Moog, "Definition of eigenvalues for a nonlinear system," in 9th IFAC Symp. Nonlinear Control Syst., Toulouse, France, 2013, pp. 600-605.

[15] R. T. O'Brien and P. A. Iglesias, "On the poles and zeros of linear, time-varying systems," IEEE Trans. Circuits Syst.: Fundamental Theory Appl., vol. 48, no. 5, pp. 565-577, 2001.

[16] K. Zenger and R. Ylinen, "Poles and zeros of multivariable linear timevarying systems," Proc. 15th IFAC World Congr., vol. 35, no. 1, pp. 261-266, 2002
[17] I. Mezić, "Spectral properties of dynamical systems, model reduction and decompositions," Nonlinear Dynamics, vol. 41, no. 1, pp. 309-325, 2005.

[18] G. I. Cirillo, A. Mauroy, L. Renson, G. Kerschen, and R. Sepulchre, "A spectral characterization of nonlinear normal modes," J. Sound Vib., vol. 377, pp. 284-301, 2016.

[19] Y. Kawano and T. Ohtsuka, "Observability analysis of nonlinear systems using pseudo-linear transformation," in 9th IFAC Symp. Nonlinear Control Syst., Toulouse, France, 2013, pp. 606-611.

[20] - "Stability criteria with nonlinear eigenvalues for diagonalizable nonlinear systems," Syst. Control Lett., vol. 86, pp. 41-47, 2015.

[21] _ - "Nonlinear eigenvalue approach to differential Riccati equations for contraction analysis," IEEE Trans. Autom. Control, vol. 62, no. 12, pp. 6497-6504, 2017

[22] A. Padoan and A. Astolfi, "Model reduction by moment matching at isolated singularities for linear systems: a geometric approach," in Proc. 56th Conf. Decision Control, Melbourne, Australia, 2017, pp. 4807-4812.

[23] — "Eigenvalues and poles of a nonlinear system: a geometric approach," in Proc. 56th Conf. Decision Control, Melbourne, Australia, 2017, pp. 2575-2580.

[24] P. Lancaster and M. Tismenetsky, The Theory of Matrices (2nd edition). New York, NY, USA: Academic, 1985.

[25] A. C. Antoulas, Approximation of large-scale dynamical systems. Philadelphia, PA, USA: SIAM, 2005.

[26] R. A. Horn and C. R. Johnson, Matrix analysis (2nd edition). Cambridge, U.K.: Cambridge Univ. Press, 1994.

[27] A. Bressan and B. Piccoli, Introduction to the mathematical theory of control. Philadelphia, PA, USA: SIAM, 2007.

[28] A. Astolfi, D. Karagiannis, and R. Ortega, Nonlinear and adaptive control with applications. London, U.K.: Springer-Verlag, 2007.

[29] J. Guckenheimer and P. Holmes, Nonlinear oscillations, dynamical systems, and bifurcations of vector fields. New York, NY, USA: SpringerVerlag, 1991.

[30] W. Respondek, "On decomposition of nonlinear control systems," Syst. Control Lett., vol. 1, no. 5, pp. 301-308, 1982.

[31] J. D. Murray, Mathematical biology I - An introduction. New York, NY, USA: Springer-Verlag, 2002.

[32] A. Isidori, A. Krener, C. Gori-Giorgi, and S. Monaco, "Nonlinear decoupling via feedback: a differential geometric approach," IEEE Trans. Autom. Control, vol. 26, no. 2, pp. 331-345, 1981.

[33] A. Padoan, G. Scarciotti, and A. Astolfi, "A geometric characterisation of the persistence of excitation condition for the solutions of autonomous systems," IEEE Trans. Autom. Control, vol. 62, no. 11, pp. 5666-5677, 2017.

[34] J. Carr, Application of center manifold theory. New York: SpringerVerlag, 1981.

[35] A. Padoan and A. Astolfi, "On the notion of moment at a pole of a nonlinear system," in Proc. 23rd Math. Symp. Netw. Syst., Hong Kong, 2018, pp. 408-413.

[36] G. Scarciotti, "Steady-state matching and model reduction for systems of differential-algebraic equations," IEEE Trans. Autom. Control, vol. 62, no. 10, pp. 5372-5379, 2017.

[37] L. Dai, Singular control systems. Berlin, Germany: Springer-Verlag, 1989.

[38] J. W. Demmel and B. Kågström, "Computing stable eigendecompositions of matrix pencils," Lin. Alg. Appl., vol. 88, pp. 139-186, 1987.

[39] B. Kågström and P. Van Dooren, "A generalized state-space approach for the additive decomposition of a transfer matrix," J. Num. Linear Alg. App., vol. 1, no. 2, pp. 165-181, 1992.

[40] J. K. Hale and S. M. V. Lunel, Introduction to functional differential equations. New York, NY, USA: Springer, 1993.

[41] A. Bensoussan, G. Da Prato, M. C. Delfour, and S. Mitter, Representation and control of infinite dimensional systems. Boston, MA, USA: Birkhäuser, 2007.

[42] D. Jordan and P. Smith, Nonlinear ordinary differential equations: an introduction for scientists and engineers (4th edition). Oxford, U.K.: Oxford Univ. Press, 2007.

[43] T. Hu, A. R. Teel, and Z. Lin, "Lyapunov characterization of forced oscillations," Automatica, vol. 41, no. 10, pp. 1723-1735, 2005.

[44] I. Kovacic and M. J. Brennan, Eds., The Duffing equation: nonlinear oscillators and their behaviour. New York, NY, USA: Wiley, 2011. 